\title{
Acute Visual Loss due to Acute Angle-Closure Glaucoma
}

\author{
Hae Ri Kim ${ }^{1}$, Kang Wook Lee ${ }^{1 *}$, Won Jung Choi ${ }^{1}$, Hong Jae Jeon ${ }^{1}$, Young Rok Ham ${ }^{1}$, Kyung nam Kim², \\ Dae Eun $\mathrm{Choi}^{1}$ and Ki Ryang $\mathrm{Na}^{1}$ \\ ${ }^{1}$ Division of Nephrology, Department of Nephrology, Chungnam National University Hospital, Daejeon, South Korea \\ ${ }^{2}$ Department of Ophthalmology, Chungnam National University Hospital, Daejeon, South Korea
}

*Corresponding author: Kang Wook Lee, Division of Nephrology, Department of Nephrology, Chungnam National University Hospital, 282 Munhwaro Junggu, Daejeon, 35015, South Korea, Tel : +82422807160; E-mail : kwlee@cnu.ac.kr

Received date: September 04, 2017; Accepted date: October 03, 2017; Published date: October 09, 2017

Citation: Hae Ri Kim, Kang Wook Lee, Won Jung Choi, Hong Jae Jeon, Young Rok Ham, et al. (2017) Acute Visual Loss due to Acute Angle-Closure Glaucoma after Kidney Transplantation. J Clin Exp Nephrol Vol.2 No.3: 47.

Copyright: (C2017 Kim HR, et al. This is an open-access article distributed under the terms of the Creative Commons Attribution License, which permits unrestricted use, distribution, and reproduction in any medium, provided the original author and source are credited.

\section{Abstract}

Acute angle-closure glaucoma is an ocular emergency and is distinct due to its acute presentation, need for immediate treatment, and well-established anatomic pathology. Although some patients develop increased intraocular pressure after kidney transplantation, few patients are diagnosed with glaucoma. Most glaucoma that appears after kidney transplantation is of the open-angle type and is probably associated with steroid treatment. Acute angleclosure glaucoma after kidney transplantation is rare. We report a case of acute angle-closure glaucoma, suggested to be steroid independent that developed after kidney transplantation and resulted in progression to permanent visual loss.

Keywords: Angle closure; Glaucoma; Kidney transplantation; Visual loss

\section{Abbreviations:}

ONH: Optic Nerve Head; RNFL: Retinal Nerve Fiber Layer; OU: Both Eyes; SUP: Superior; NAS: Nasal; TEMP: Temporal; OD: Right Eye; OS: Left Eye; C/D: Cup to Disc

\section{Introduction}

Glaucoma is an optic neuropathy than can cause optic nerve damage and loss of vision [1]. Although some studies have shown that $10 \%$ of patients develop increased intraocular pressure (IOP) after kidney transplantation (KT), glaucoma is diagnosed in few patients [2].

Although acute angle-closure glaucoma is very rare, it is an ocular emergency that produces critical complications, including permanent visual loss. Kopsa et al. reported a case of corticosteroid-induced glaucoma that developed in both of a patient's eyes after management of an acute rejection episode from KT [3]. IOP tends to increase slowly in cases of steroidrelated glaucoma, which usually appears as primary open-angle glaucoma, but can also appear as low-tension glaucoma [4].

Acute angle-closure glaucoma appearing shortly after KT is rare. Here, we describe the case of an older woman who developed acute angle-closure glaucoma (suggested to be steroid independent) after deceased-donor $\mathrm{KT}$, resulting in progression to permanent visual loss.

\section{Case Description}

A 65-year-old woman was admitted to our hospital for cadaveric KT. She had been diagnosed with mesangial proliferative glomerulonephritis 15 years previously, and her kidney function had decreased gradually. Peritoneal dialysis had been started because of end-stage renal failure 10 years previously. The patient had no history of diabetes. She was taking antihypertensive medicines, such as barnidipine and candesartan. Cinacalcet was prescribed to control secondary hyperparathyroidism.

Basiliximab was injected just before KT and 4 days after surgery as induction immunosuppressive therapy. Tacrolimus, mycophenolate, and a high-dose glucocorticoid were started as the main immunosuppressive therapy. Target trough levels of tacrolimus were $10-12 \mathrm{ng} / \mathrm{dL}$. The patient's urine output increased to $150-400 \mathrm{~mL} / \mathrm{h}$ shortly after KT. The results of transplanted kidney Doppler ultrasonography and a Tc-99m DTPA scan were normal. Her serum creatinine level was normalized 5 days after surgery, and urine output was maintained well.

The patient complained of discomfort and an irritating sensation in her right eye on postoperative day 3. An ophthalmologist detected a corneal abrasion on the patient's right eye and a cataract in the left eye. Artificial tears eye drops were prescribed for the corneal abrasion. At that time, the patient's IOP was at the upper limit of normal in both eyes (left, $21 \mathrm{mmHg}$; right, $22 \mathrm{mmHg}$ ). Uncorrected visual acuity was 0.3 
and the anterior chamber was shallow in both eyes. Relative afferent pupillary defect (RAPD) is a condition in which the pupils respond differently to a light stimulus shone in one eye at a time due to unilateral or asymmetric disease of the retina or optic nerve. No RAPD was seen at that time.

The patient's symptoms subsided shortly after administration of the artificial tears eye drops. However, she experienced severe pain in both eyes and headache $5 \mathrm{~h}$ later. Tramadol and acetaminophen were administered. The eye pain and headache were relieved, but had not fully subsided, on postoperative day 4. The patient complained of mild headache and nausea on postoperative days 6 and 8 . Acetaminophen and anti-emetics were prescribed.
The patient complained of left eye discomfort and pain on postoperative day 12 . In addition, new blurred vision occurred. We consulted the ophthalmology department again. A vision test showed that the left eye had no light sense. A test for RAPD was positive in the left eye. The IOP was very high in the left eye (52 $\mathrm{mmHg}$; right eye, $15 \mathrm{mmHg}$ ). The cup to disc (C/D) ratio was not measurable due to severe disc edema on funduscopy. The patient's vision was almost completely lost in the left eye, which was thought to be due to the elevated IOP. The accurate measurement of disc size via funduscopy was difficult due to severe edema at that time. However, the cup was enlarged (Figure 1).
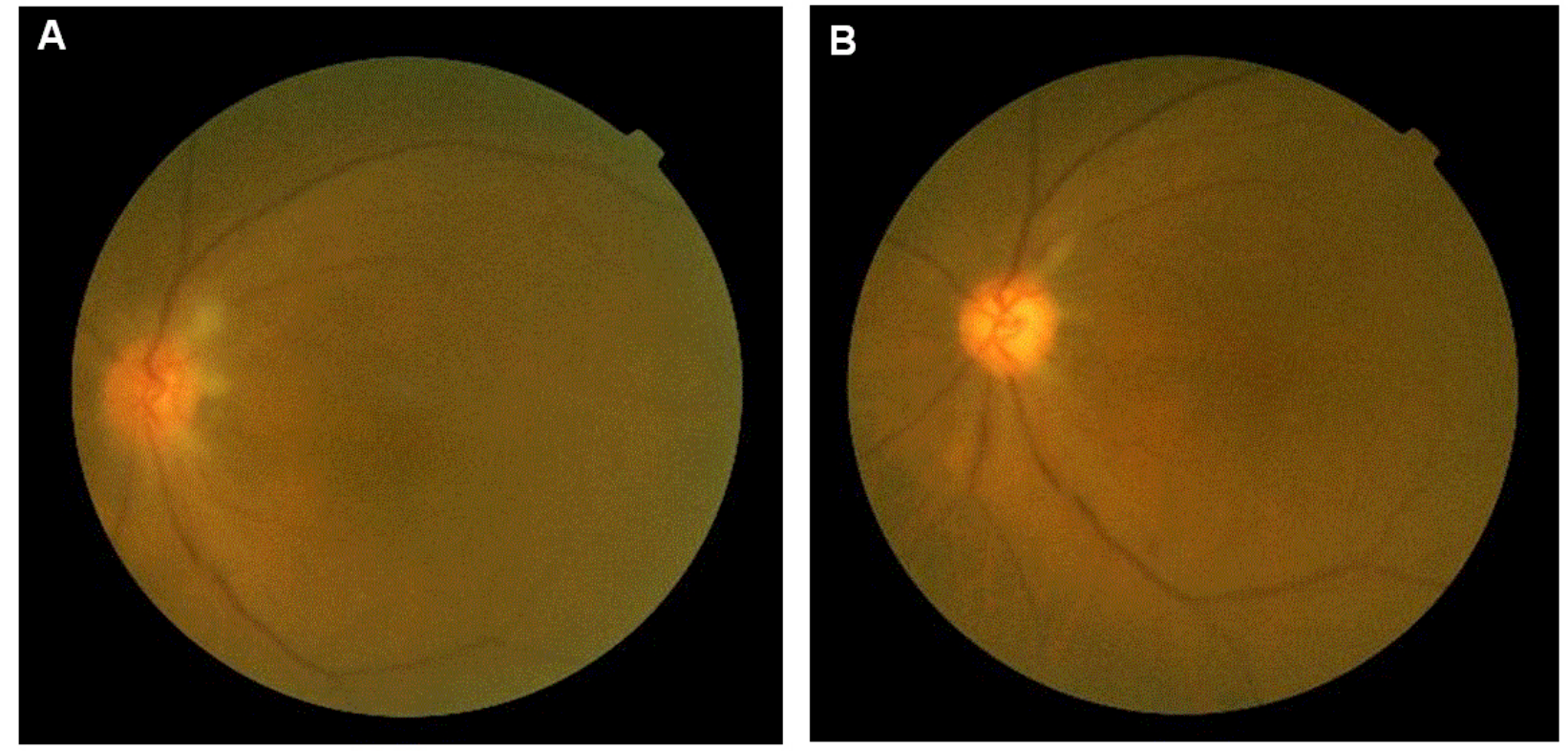

Figure 1: Representative funduscopic photographs. (A) Optic disc edema, suggesting increased intraocular pressure, is visible. (B) The disc edema persisted post-iridotomy.

The patient was diagnosed with acute closed-angle glaucoma, optic nerve damage, and vitreous hemorrhage. Mannitol was administered to control the IOP, and the left eye pain subsided. However, the patient's visual loss continued, and a poor prognosis was predicted.

The IOP of the left eye was well controlled on postoperative day 15 . However, the vision loss and pain continued in the left eye. The patient underwent emergency iridotomy, which markedly relieved her left eye pain.

The patient underwent left post-chamber lensectomy on postoperative day 23 to control repeated severe pain in the left eye. The disc edema had decreased, but persisted at the time of follow-up funduscopic examination. Accurate measurement of disc size was difficult because of the edema. However, we assumed that the $C / D$ ratio had increased above the normal range because the cup had enlarged.

Optical coherence tomography (OCT) was performed 4 weeks after discharge to evaluate the optic nerve and the thickness of the nerve fiber layer. OCT showed that the left eye had a decreased rim area and increased $C / D$ ratio $(0.86)$. The $C / D$ ratio in the right eye was normal. The retinal nerve fiber layer was generally thin in the left eye, especially on the superior and inferior sides. The OCT results revealed optic nerve damage to the left eye (Figure 2). 

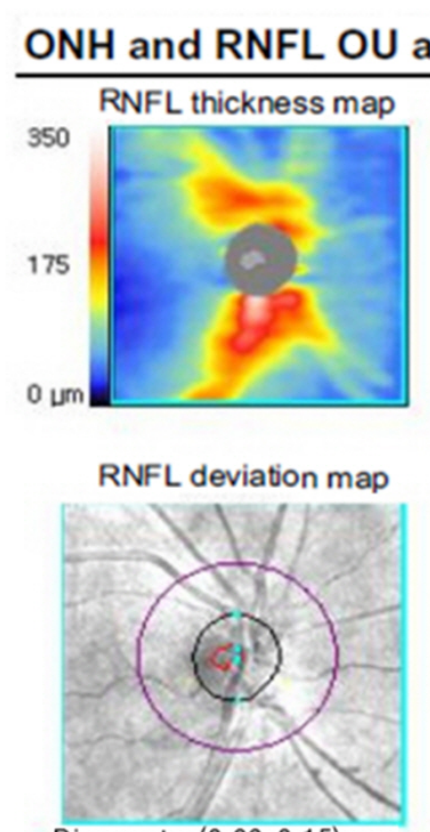

Disc center $(0.03 .0 .15) \mathrm{mm}$

\begin{tabular}{|r|c|c|}
\hline & OD & OS \\
\hline Average RNFL thickness & $110 \mu \mathrm{m}$ & $76 \mu \mathrm{m}$ \\
\hline RNFL summary & \multicolumn{2}{|c|}{$54 \%$} \\
\hline Rim area & $1.00 \mathrm{~mm}^{2}$ & $0.54 \mathrm{~mm}^{2}$ \\
\hline Disc area & $1.84 \mathrm{~mm}^{2}$ & $2.02 \mathrm{~mm}^{2}$ \\
\hline Average CD rafo & 0.28 & 0.86 \\
\hline Vertical CD ratio & 0.25 & 0.70 \\
\hline Cup volume & $0.019 \mathrm{~mm}^{3}$ & $0.010 \mathrm{~mm}^{3}$ \\
\hline
\end{tabular}

Neuro-retinal rim thickness

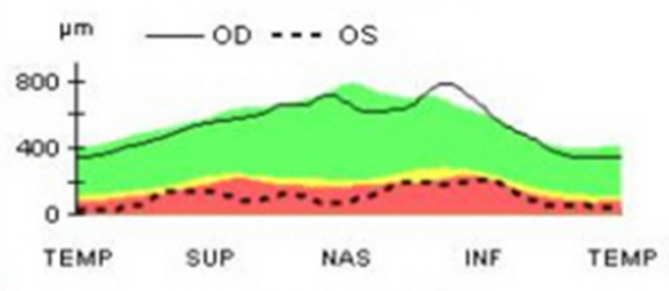

RNFL thickness

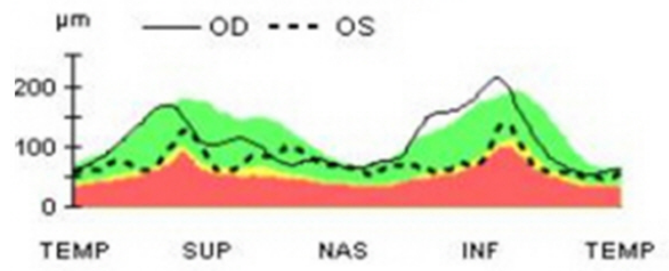

OD $\bigcirc$ OS

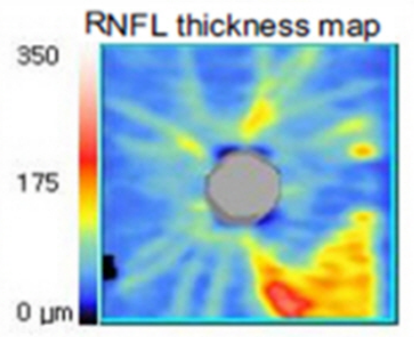

RNFL deviation map

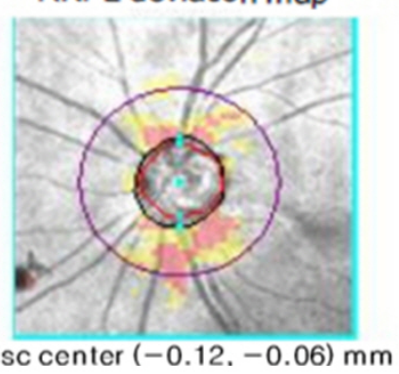

Figure 2: Optical coherence tomography. The C/D ratio increased in the left eye to 0.86 and in the right eye to 0.28 . The RNFL was generally thinner than normal in both eyes. It was especially thin on the superior and inferior sides of the left eye, suggesting glaucomatic optic nerve damage.

Right lensectomy was performed 5 weeks after discharge to prevent an acute glaucoma attack because the right eye also had shallow depth.

\section{Discussion}

Glaucoma is an optic neuropathy caused by compression of the optic nerve or impairment of the blood supply due to increased IOP that can cause visual field loss and decreased vision [1]. Glaucoma can be classified as angle-closure or openangle glaucoma. In this case, increased posterior pressure forced the iris to migrate to the cornea, resulting in a compressed anterior angle and closed-angle glaucoma. The anterior angle is created from the back of the iris to the front of the cornea, and the aqueous humor drainage pathway is blocked when the anterior angle is compressed and IOP increases rapidly.

In our case, consideration of the patient's symptoms and examination resulted in a diagnosis of acute angle-closure glaucoma. The patient developed angle-closure symptoms of headache, eye pain, and blurred vision, as well as increased IOP. Narrowing of the anterior chamber depth was seen via slit-lamp bio microscopy. The $C / D$ ratio was difficult to measure, but we assumed that the optic nerve had been damaged because of the enlarged cup. The C/D ratio is the ratio of the cup to the optic disc. The cup is a sink region at the head of the optic nerve in the center of the optic disc. In glaucoma, increased IOP decreases blood flow to the optic nerve, which destroys the nerve. As glaucoma progresses, the $C / D$ ratio increases. When the $C / D$ ratio is $>0.8$, glaucoma is suspected.

Old age, family history, female sex, a shallow anterior chamber, and increased IOP are known risk factors for acute glaucoma $[5,6]$. Lin et al. reported a mean anterior chamber depth of $2.7 \mathrm{~mm}$ in a patient with acute angle-closure glaucoma [7]. In our case, old age, female sex, and small initial anterior chamber depths (left eye, $1.5 \mathrm{~mm}$; right eye, $3.0 \mathrm{~mm}$ ) were among the risk factors. Considering these factors, our patient was at greater risk of glaucoma relative to other patients.

Gayat at al. noted that acute angle-closure glaucoma is related to general anesthesia. He reported on a patient with decreased visual acuity who complained of eye pain and nausea 1 day after surgery [8].

In our case, the IOP was in the normal range 3 days after KT. Because the patient's symptoms were aggravated beginning 3 
days after general anaesthesia, we believe that the glaucoma was not likely caused by the surgery or medication prescribed after surgery.

Drugs can cause glaucoma. latrogenic glaucoma can occur secondary to drug administration and can result in blindness due to optic nerve damage caused by increased IOP [9]. Adrenergic agents, anticholinergics, antihistamines, and antidepressants are known to cause acute closed-angle glaucoma $[10,11]$. Steroids can also increase the incidence of glaucoma $[9,12]$.

Ticho et al. reported on the development of steroid-induced glaucoma, resulting in disk cupping, visual field loss, and blindness, after KT [13]. IOP tends to increase slowly in cases of steroid-related glaucoma, which usually appears as primary open-angle glaucoma, but can also appear as low-tension glaucoma $[12,14,15]$.

In our case, an acute attack of angle-closure glaucoma occurred on one side. The IOP was very high, and the patient's symptoms were acute. These features differ from those of steroid-induced glaucoma. The patient's risk factors (old age, female sex, and a shallow anterior chamber) and the administration of steroids may have increased the risk of acute glaucoma. In particular, the severely shallow anterior chamber in the left eye may have been an important cause of the glaucoma attack in the present case.

Most glaucoma attacks are seen when pupillary block occurs in a patient unaware of their narrow iridocorneal angle $[9,16]$. Iridotomy is the most effective approach to decrease pressure in the early stages of the disease $[9,16]$. However, it is not effective when a patient has had vitreous hemorrhage or has ciliary or suprachoroidal effusion $[9,16]$. In our case, we believed that iridotomy might not be effective because of vitreous haemorrhage.

Aung at al. reported on long-term outcomes of acute primary angle closure in 90 Asians followed for 4-10 years after the diagnosis of this condition. They observed glaucoma in $47.8 \%$ $(n=43)$ of cases, glaucomatous optic neuropathy and compatible visual field loss in $42.2 \%(n=38)$ of cases, and blindness in $17.8 \%$ $(n=16)$ of cases. About $50 \%$ of the blindness cases were caused by glaucoma. In our case, the patient suffered permanent visual loss in the left eye [16].

In summary, we report a case of acute angle-closure glaucoma resulting in permanent visual loss shortly after KT in a 65-yearold woman. Because of the detection of corneal abrasion and normal IOP on the initial ophthalmic examination, glaucoma was not considered, despite the intermittent occurrence of ocular symptoms. The general anaesthesia and drugs used during KT constitute risk factors for increased IOP. Selective ophthalmologic screening may be needed for patients with risk factors, such as older age, family history, and ocular symptoms, before KT.

\section{References}

1. Weinreb RN, Aung T, Medeiros FA (2014) The pathophysiology and treatment of glaucoma: a review. Jama 311: 1901-1911.

2. Adhikary H, Sells R, Basu P (1982) Ocular complications of systemic steroid after renal transplantation and their association with HLA. Br J Ophthalmol 66: 290-291.

3. Kopsa H, Bettelheim H, Schmidt P, Zazgornik J (1974) Steroid glaucoma after renal transplantation (author's transl). Deutsche medizinische Wochenschrift 99: 576.

4. Dada T, Nair S, Dhawan M (2009) Steroid-induced glaucoma. J Curr Glau Practice 3: 33-38.

5. Jones III R, Rhee DJ (2006) Corticosteroid-induced ocular hypertension and glaucoma: a brief review and update of the literature. Curr Opin Ophthalmol 17: 163-167.

6. Kersey J, Broadway D (2006) Corticosteroid-induced glaucoma: a review of the literature. Eye 20: 407-416.

7. Lin Y, Wang T, Hung P (1997) Biometric study of acute primary angle-closure glaucoma. J Formos Med Assoc 96: 908-912.

8. Gayat E, Gabison E, Devys J-M (2011) Bilateral angle closure glaucoma after general anesthesia. Anesth Analg 112: 126-128.

9. Lachkar Y, Bouassida W (2007) Drug-induced acute angle closure glaucoma. Curr Opin Ophthalmol 18: 129-133.

10. Subak-Sharpe I, Low S, Nolan W, Foster PJ (2010) Pharmacological and environmental factors in primary angle-closure glaucoma. $\mathrm{Br}$ Med Bull 93: 125-143.

11. Ah-kee EY, Egong E, Shafi A, Lim LT, Yim JL (2015) A review of drug induced acute angle closure glaucoma for non-ophthalmologists. Qatar Med J 10: 6

12. Razeghinejad MR, Myers JS, Katz LJ (2011) latrogenic glaucoma secondary to medications. Am J Med 124: 20-25.

13. Ticho U, Durst A, Licht A, Berkowitz S (1977) Steroid-induced glaucoma and cataract in renal transplant recipients. Isr J Med Sci 13: 871-874.

14. Tripathi RC, Parapuram SK, Tripathi BJ, Zhong Y, Chalam K (1999) Corticosteroids and glaucoma risk. Drugs Aging 15: 439-450.

15. Razeghinejad MR, Katz LJ (2011) Steroid-induced iatrogenic glaucoma. Ophthalmic Res 47: 66-80.

16. Aung T, Friedman DS, Chew PT, Ang LP, Gazzard G, et al. (2004) Long-term outcomes in Asians after acute primary angle closure. Ophthalmology 111: 1464-1469. 